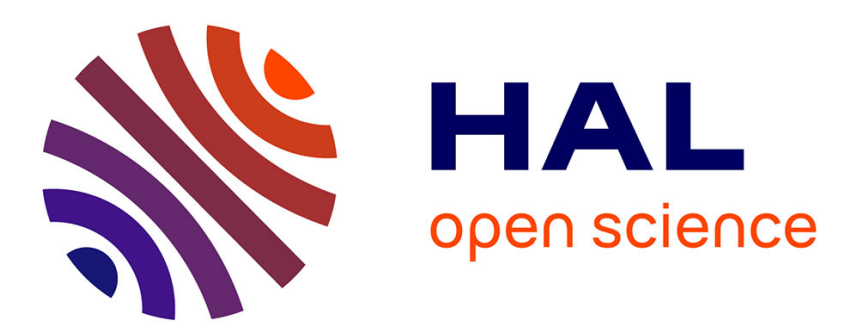

\title{
A Versatile Computational Strategy To Characterize the Free-Energy Landscape of Excited States in Oligofluorenes
}

\author{
Concetta Cozza, Massimiliano Bonomi, Adriana Pietropaolo
}

\section{- To cite this version:}

Concetta Cozza, Massimiliano Bonomi, Adriana Pietropaolo. A Versatile Computational Strategy To Characterize the Free-Energy Landscape of Excited States in Oligofluorenes. Journal of Chemical Theory and Computation, 2018, 14 (11), pp.5441-5445. 10.1021/acs.jctc.8b00949 . hal-03136519

\author{
HAL Id: hal-03136519 \\ https://hal.science/hal-03136519
}

Submitted on 9 Feb 2021

HAL is a multi-disciplinary open access archive for the deposit and dissemination of scientific research documents, whether they are published or not. The documents may come from teaching and research institutions in France or abroad, or from public or private research centers.
L'archive ouverte pluridisciplinaire HAL, est destinée au dépôt et à la diffusion de documents scientifiques de niveau recherche, publiés ou non, émanant des établissements d'enseignement et de recherche français ou étrangers, des laboratoires publics ou privés. 


\title{
A versatile computational strategy to characterize the free-energy landscape of excited states in oligofluorenes
}

\author{
Concetta Cozza ${ }^{1}$, Massimiliano Bonomi ${ }^{2 *}$, Adriana Pietropaolo ${ }^{1 *}$ \\ ${ }^{1}$ Dipartimento di Scienze della Salute, Università di Catanzaro, Viale Europa, 88100 Catanzaro, Italy \\ ${ }^{2}$ Department of Chemistry, University of Cambridge, Lensfield Road, Cambridge CB2 1EW, United Kingdom \\ †Current address: Structural Bioinformatics Unit, Institut Pasteur, CNRS UMR 3528, 75015 Paris, France
}

Supporting Information Placeholder

\begin{abstract}
The $\pi$-rich rings of conjugated polymers and molecular rotors shape their typical properties, allowing a variety of chemical and photoresponsive phenomena. Herein, we present a computational method in the framework of classical simulations to estimate the free-energy gap between ground and excited states of oligofluorenes, a class of molecular rotors widely used in optoelectronic devices due to the inner torsional rotation triggered by light irradiation. We devised multiple sets of free-energy simulations in combination with free-energy perturbation theory to predict the freeenergy gap between the ground and the first excited state. The computed excitation energies show good agreement with experiments. The approach herein presented allows to achieve at the same time comprehensive sampling of the conformational landscape and accurate estimates of the excited state free-energy landscapes.
\end{abstract}

In recent years, conjugated polymers have attracted a wide attention as organic semiconductors ${ }^{1}$ for their use in devices such as organic light-emitting diodes (OLEDs), ${ }^{2}$ solar cells as well as field-effect transistors (FETs). ${ }^{3}$ Among the class of conjugated polymers, polyor oligo-fluorene derivatives are of wide interest in organic electronics, owing to their light-emitting properties. $^{4}$

A peculiar structural feature of the poly- or oligofluorene derivatives is the presence of a main-chain molecular rotor between two bridging biphenyl units (Scheme 1). This chemical arrangement allows a specific molecular response through light stimuli irradiation. ${ }^{5-10}$ For instance, the extent of a coplanar orientation of the adjacent fluorene units was observed to largely affect the electronic and optical properties. ${ }^{11}$

A wealth of computational contributions have indeed reported through the years on the structural properties of polyfluorenes, ${ }^{12}$ on their electronic structure, ${ }^{13}$ their self- assembling features, ${ }^{14}$ as well as their charge-transport 15,16 and optical properties. ${ }^{17}$ An ultra-fast torsional relaxation was achieved in oligofluorenes based on penta-(9,9-dioctylfluorene) through a sub- $150 \mathrm{fs} 3.18 \mathrm{eV}$ pump, bringing the system to the first singlet excited state (S1) and thereby pushing the molecule with a second pulse to higher excited states, ${ }^{18}$ disclosing the flattening mechanism within non-adiabatic excited state dynamics based on a mixed quantum-semiempirical approach. ${ }^{19,20}$

Excited state properties can be studied by means of excited-state ab-initio dynamics, ${ }^{21-24}$ with non adiabatic schemes $^{25-29}$ or surface-hopping based techniques, ${ }^{30-34}$ focusing a particular attention in reaching sampling acceleration in excited states. ${ }^{35-37}$ Although these methods are highly accurate, for large systems they still require a substantial amount of CPU time and memory, which ultimately limit their predictive power to only selected portions of the molecules.

Recently, enhanced sampling free-energy simulations of fluorene-based polymers in the ground-state have been reported, ${ }^{38}$ predicting the helix-sense switching from achiral racemic states to the chiral helix states, switching from random twist- to all-positive or -negative twist dihedrals. This stepwise achiral to chiral transition was described through a special purpose chiral path collective variables. ${ }^{39}$

In this framework, an estimate of free-energy differences in excited states and conformational sampling in fluorene-based polymers can boost the efforts in the realization of bespoke materials and devices with tunable optoelectronic properties. A rational design of these materials can be achieved from the knowledge of their photoresponsive properties, ${ }^{40,41}$ which depend on the excited state free-energy landscape as well as on the height of free-energy barriers and freeenergy gaps between the ground and excited states. Therefore, disclosing and predicting the structural properties and energetics of the excited states of light- 
switching molecular rotors can offer important information for the development of new functional polymers of optoelectronic interest.

In this letter we report a straightforward strategy to estimate the excited state free-energy profiles from the knowledge of the free-energy landscape of the ground state. We assessed the method by estimating the freeenergy gap between the ground and the first excited state of a fluorene pentamer, while reconstructing the related free-energy profiles. Indeed, the method can be extended to substituted polymers with alkyl chain at 9-position, generally used to improve solubility. The torsional mechanism occurring in fluorene oligomers triggered by light irradiation is described in Scheme 1, where a twistcoplanar transition is schematically shown. The rotation of a dihedral unit can direct the rest of the polymer chain, akin to the "sergeants and soldiers" principle. ${ }^{43,44}$ We therefore simulated a stepwise dihedral rotation using the first, second and third fluorene-fluorene dihedral reconstructing one-dimensional free-energy profiles.

Let us consider the Free-energy $F(s)$ as a function of one variable $s$, that in our case is the fluorene-fluorene dihedral and is used to discriminate among different rotational conformers:

$$
F(s)=\frac{-1}{\beta} \ln \frac{\int \delta(s-s(\boldsymbol{r})) e^{-\boldsymbol{\beta} \boldsymbol{V}(\boldsymbol{r})} d \boldsymbol{r}}{\int e^{-\beta V(\boldsymbol{r})} d \boldsymbol{r}}
$$

in which $V(\boldsymbol{r})$ is the interaction potential, $\mathbf{r}$ are the atomic coordinates and $\beta$ is the inverse of the $\mathrm{K}_{\mathrm{b}} \mathrm{T}$ term, where $K_{b}$ is the Boltzmann constant and $T$ is the absolute temperature of the system. We now add to $V(\boldsymbol{r})$ a Ryckaert-Bellemans torsional potential defined for a given $S_{i}$ electronic state along the $\varphi$ fluorene-fluorene dihedral, which can be described by a series of cosine functions reported in eq. 2 (additional details are reported in the Supplementary information):

$V^{S_{i}}(\varphi)=\sum_{i=0}^{5} C_{n}{ }^{S_{i}}(\cos (\varphi-180))^{n}$

The coefficients $C_{n}^{S i}$ (Tables S1,S2) were derived from the torsional potential energy scans of the singlet ground state and the first singlet excited state $\mathrm{S} 1$ of 2,2'difluorenyl at the B97D3/6-31G* and the B3LYP/6-31G* level of theories (Tables S3, S4 of Supporting Information) in the Time Dependent Density functional Theory (TDDFT) framework, which was shown to be appropriate for predicting excitation energies of organic molecules. ${ }^{44}$



Scheme 1. The mechanism of intermolecular rotation occurring in fluorene oligomers triggered by light irradiation, allowing a trans-cis isomerization.

We define $F^{S 0}(\varphi)$ the free-energy surface for the modified potential $V(\boldsymbol{r})+V^{S 0}(\varphi)$ given by equation 3 ), where $V^{S 0}(\varphi)$ is the torsional potential in the S0 ground state (eq. 2).

$F^{S 0}(\varphi)=\frac{-1}{\beta} \ln \frac{\int \delta(\varphi-\varphi(r)) e^{-\beta\left(V(r)+V^{S 0}(\varphi(r))\right.} d r}{\int e^{-\beta\left(V(r)+V^{S 0}(\varphi(r))\right.} d r}$

Analogously, we can define the free-energy surface $F^{S l}(\varphi)$, as the free-energy surface for the modified potential $V(\boldsymbol{r})+$ $V^{S l}(\varphi)$ where $V^{S l}(\varphi)$ is the torsional potential in the $S 1$ excited state (eq. 2). The two free-energy surfaces are therefore straightforwardly related each other under eq. 4:

$F^{s 1}(\varphi)=F^{s 0}(\varphi)+V^{S 1}(\varphi)-V^{S 0}(\varphi)+c \quad 4$

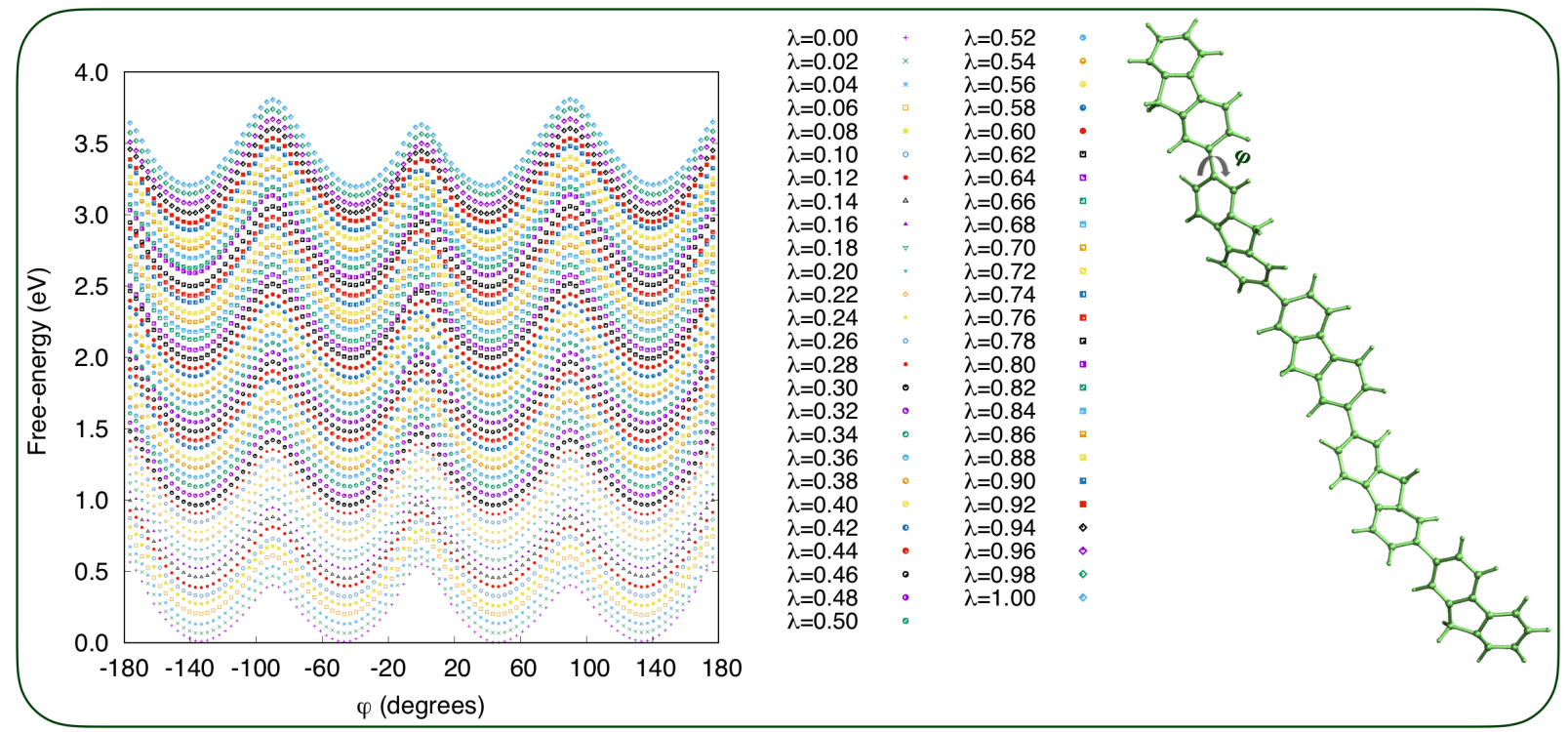

Figure 1. Free-energy profiles of the S0-S1 excitation in a fluorene pentamer obtained through FEP simulations in the welltempered metadynamics framework as a function of the dihedral rotation, $\varphi$. The torsional potential is obtained through a potential energy scan at the B97D3/6-31G* level of theory. The $\lambda$ parameter ranges from 0 to 1 , identifying the S0 and S1 states with a spacing of 0.02 , in order to allow the overlap of the free-energy profiles. 
In order to evaluate the free-energy difference between S0 and S1 states, we used the free-energy perturbation (FEP) theory. We introduced multiple intermediate windows between S0 and S1 through a control parameter, $\lambda$, which ranges from 0 to 1 . The external potential acting on each state $\lambda$ is described by:

$$
V_{\lambda}(\varphi)=(1-\lambda) V^{S 0}+\lambda V^{S 1}
$$

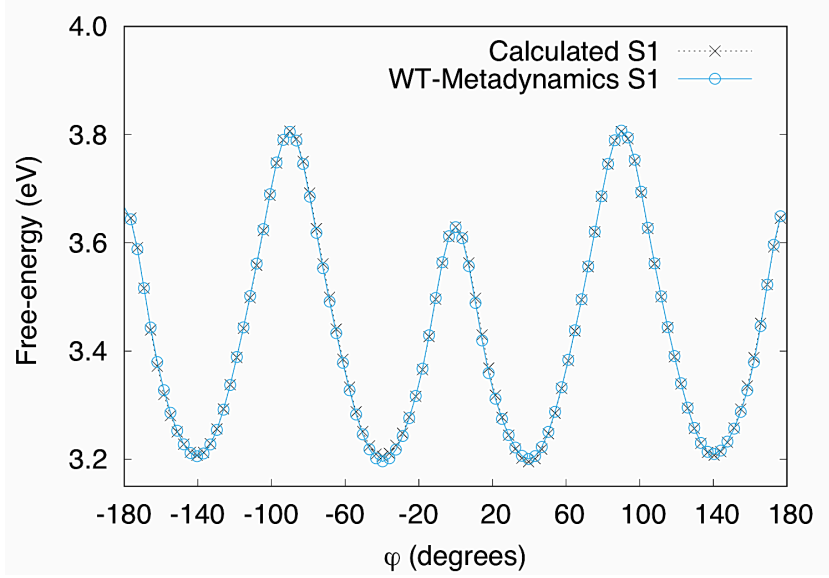

Figure 2. Free-energy profiles of the S1 state of the fluorene pentamer as a function of the dihedral rotation, $\varphi$, obtained from the well-tempered metadynamics simulation in the S1 state (blue dot line) and calculated from the free-energy profile of the ground state (black cross line) using eq. 4. The torsional potential is obtained through a potential energy scan within the B97D3/6-31G* level of theory.

A step-size $\Delta \lambda$ of 0.02 was chosen in order to obtain an overlap between the energy distributions of neighboring windows. As external potential $\mathrm{V}_{\lambda}(\varphi)$, we used the one parametrized at the B97D3/6-31G* and B3LYP/6-31G* levels of theory (Figure 1, Figures S1-S2 of the Supporting Information). Well-tempered ${ }^{45}$ metadynamics $^{46}$ using the $\varphi$ torsion as collective variable (Figure 1) were run in combination with FEP to ensure exhaustive configurational sampling and reconstruct the free-energy landscape as a function of the fluorene-fluorene dihedral.

We estimated the S0-S1 free-energy gap as the sum over the free-energy differences between neighboring windows using eq. 6 :

$$
\begin{aligned}
& \Delta F^{S 0 \rightarrow S 1}=F^{s 1}-F^{s 0}=\sum_{i} \Delta F^{\lambda_{i}+\Delta \lambda \rightarrow \lambda_{i}} \\
& \Delta F^{\lambda_{i}+\Delta \lambda \rightarrow \lambda_{i}}=-\frac{1}{\beta} \log \left\langle e^{-\beta\left(E_{\lambda_{i}}-E_{\lambda_{i}+\Delta \lambda}\right)}\right\rangle
\end{aligned}
$$

The ensemble average in eq. 7 was calculated after removing the effect of the metadynamics bias potential $^{47}$

We tested the method also on the inner torsions of the pentafluorene (i.e. the second and the third) and no significant differences were observed in the calculations of the excitation energies among the pentamer torsions (Figures S1-S6, additional details are reported in the Supporting Information). The calculated S0-S1 free-energy gap along each torsion of the pentafluorene under the B97D3/6-31G* is $3.196 \mathrm{eV}$ with a statistical error of $2 \times 10^{-5} \mathrm{eV}$ and $3.568 \mathrm{eV}$ under the B3LYP/6-31G* with a statistical error of $5 \times 10^{-5} \mathrm{eV}$ (Figures S7-S12, details on the error block analysis for the FEP simulations are reported in the Supporting Information). These predictions, particularly the one with the B97D3/6$31 \mathrm{G}^{*}$ parametrization show a good agreement with the experimental excitation energy of S0-S1 transition allowed through a pump pulse of $3.18 \mathrm{eV},{ }^{17}$ reaching an accuracy of $0.5 \%$ for the FEP simulations using the B97D3 functional. Furthermore, the free-energy profiles calculated from the ground state under eq. 4 and those reconstructed from well-tempered metadynamics/FEP simulations in the S1 state feature a good superimposition (Figure 2). They both show a decrease in the free-energy barrier in the coplanar state of $0.1 \mathrm{eV}$ with respect to that belong to the ground state (Figure 3), typical of a twisted-coplanar transition of biphenyl-based rotors. ${ }^{48}$ The free-energy profiles of S0 and S1 states are reported in Figure 3, together with the Franck-Condon (FC) vertical excitations. This latter is higher 
in energy of $0.1 \mathrm{eV}$ with respect to the free-energy profile of the $\mathrm{S} 1$ state where the $\mathrm{V}^{\mathrm{S} 1}(\varphi)$ potential was derived through a relaxed potential energy surface scan.

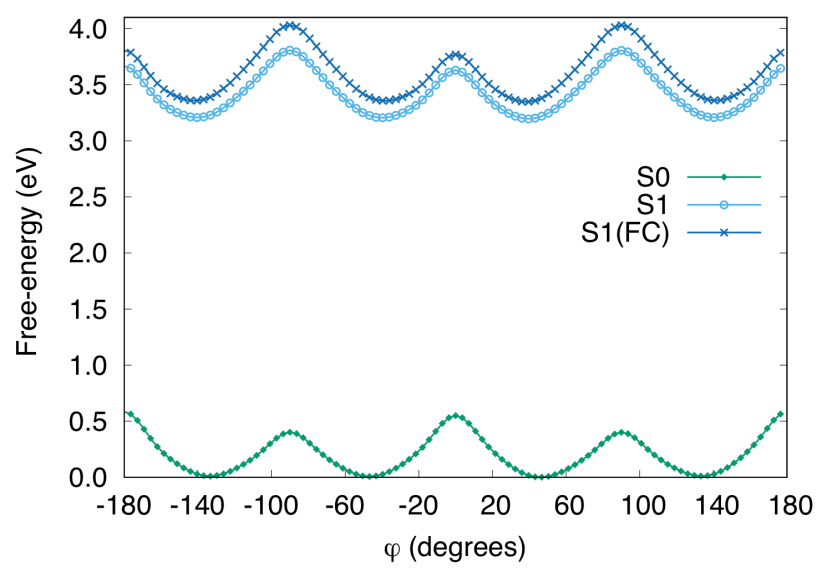

Figure 3. Free-energy profiles of the fluorene pentamer as a function of the dihedral rotation, $\varphi$ parametrized at the B97D3/6-31G* level of theory, in the S0 state reconstructed from a well-tempered metadynamics simulation (green-diamond line) and in S1 state (cyan-dot line) calculated using eq. 4 of the main text. The free-energy profile in the S1 state reached through a Franck-Condon (FC) vertical transition from the ground state is also shown (blue-cross line).

It is clear that the efficiency of the method is dependent on the ab-initio torsional potential, which determines the accuracy of the excited state free-energy surface and excited state sampling. The accuracy of the FEP simulations depends on the number of the $\lambda$-windows, which is determined by requiring sufficient overlaps between the energy distributions in neighboring $\lambda$-windows. Therefore, the computational cost scales linearly with the number of windows. In these regards, the free-energy differences of S0-S1 states calculated through FEP simulations closely match the ab-initio S0-S1 internal energy gap calculated as the difference of the internal energies of S0 and $\mathrm{S} 1$ states at 37 degrees $(3.16 \mathrm{eV}$ for B97D3/6-31G* and 3.54 for B3LYP/6-31G*), corresponding to the absolute minimum of the ground state PES. In the fluorene pentamer, it is therefore possible to consider as integration constant in eq. 4 the ab-initio S0-S1 internal energy gap estimate, conducting only one free-energy simulation in the ground-state without additional FEP simulations and reducing the computational cost to that of a single well-tempered metadynamics simulation.

Indeed, the choice of appropriate sets of functional and basis sets allow a general extension of the method to a variety of molecular and macromolecular rotors, affording large-scale transitions in excited states. Nevertheless, free-energy perturbation theory in combination with well-tempered metadynamics allows a convergence of the free-energy surfaces of the ground and excited states, predicting the morphologies and energetics of the excited state and ground-state conformations. Although the proposed approach cannot estimate excited state dynamics or decays among the electronic states, it allows energetics predictions and sampling of large-scale molecular systems in their excited states that for standard ab-initio simulations are so far restricted to hundred of atoms. Moreover, large-scale fluctuations can occur from nanosecond to microseconds, a time-scale that can be accessible with this enhanced sampling scheme, keeping a comparable accuracy in the excitation energy estimates with TD-DFT calculations, owing to the calculation of the internal energy gap at the TD-DFT level. The presented approach will therefore enable predicting the properties of excited state conformations of large molecules, long polymer chains or aggregates using classical simulations. Indeed, the conformations sampled in excited states with this approach can be also used to speed up the convergence of excited state ab-initio simulations.

In summary, a method to calculate the free-energy gap between ground and excited states, with enhanced sampling of their relevant conformations in the framework of classical simulations is herein reported. The presented approach can be used to reconstruct the energetics in the excited states of light-switchable polymers, combining advanced sampling methods with free-energy perturbation theory and using an excited state torsional potential. The properties of photoactive 
polymers with rotable groups, where specific polymer sections rotate through light-stimuli, can be predicted in terms of free-energy barriers, conformational sampling and free-energy gaps between ground and excited states.

\section{ASSOCIATED CONTENT}

\section{Supporting Information.}

Computational details, tables of the coefficients of the Ryckaert-Bellemans torsional potential, tables of the potential energy scans and figures. This material is available free of charge on the ACS Publications website.

\section{AUTHOR INFORMATION}

\section{Corresponding Author}

*apietropaolo@unicz.it; massimiliano.bonomi@pasteur.fr

Notes

The authors declare no competing financial interest.

\section{Funding Sources}

ISCRA, MIUR- PRIN20157WZM8

\section{ACKNOWLEDGMENT}

The ISCRA supercomputing initiative and the Italian Ministry of University and Research (MIUR PRIN20157WZM8) are acknowledged for computational resources and financial support respectively.

\section{REFERENCES}

(1) Noriega, R.; Rivnay, J.; Vandewal, K; Koch, F. P. V.; Stingelin, N; Smith, P; Toney, M. F.; Salleo A. A general relationship between disorder, aggregation and charge transport in conjugated polymers. Nat. Mater. 2013, 12, 10381044.

(2) Grimsdale, A. C.; Chan, K. L. ; Martin, R. E. ; Jokisz, P. G.; Holmes, A. B. Synthesis of light-emitting conjugated polymers for applications in electroluminescent devices. Chem Rev. 2009, 109, 897-1091.

(3) Facchetti, A. $\pi$-conjugated polymers for organic electronics and photovoltaic cell applications. Chem. Mater. 2011, $23,733-758$.

(4) Scherf, U.; List E.J.W. Semiconducting polyfluorenes-towards reliable structure-property relationships. $A d v$. Mater. 2002, 14, 477-487.

(5) Wang, Y.; Sakamoto, T.; Nakano, T. Molecular chirality induction to an achiral $\pi$-conjugated polymer by circularly polarized light. Chem. Commun. 2012, 48, 1871-1873.

(6) Y. Wang, T. Harada, L. Q. Phuong, Y. Kanemitsu, T. Nakano Helix Induction to Polyfluorenes Using Circularly Polarized Light: Chirality Amplification, Phase-Selective Induction, and Anisotropic Emission. Macromolecules 2018, 51, 17, 6865-6877.

(7) Pietropaolo, A.; Nakano, T. Molecular Mechanism of Polyacrylate Helix Sense Switching across Its Free Energy Landscape. J. Am. Chem. Soc. 2013, 135, 5509-5512.

(8) Pietropaolo ,A.; Tang, S.; Raymo, F.M. Free-energy predictions and absorption spectra calculations for supramolecular nanocarriers and their photoactive cargo. Nanoscale 2017, 9, 4989-4994.

(9) Angiolini, L; Giorgini, L; Li, H; Golemme, A; Mauriello, F; Termine, R. Synthesis, characterization and photoconductive properties of optically active methacrylic polymers bearing side chain 9-phenylcarbazole moieties. Polymer 2010, 51, 368-377.

(10) Angiolini, L; Benelli, T; Giorgini, L; Mauriello, F; Salatelli, E; Bozio, R; Daurù, A, Pedron, D. Synthesis, chiroptical properties and photoinduced birefringence of optically active methacrylic copolymers bearing side-chain bisazoaromatic moieties. Eur. Polym. J. 2007, 43, 3550-3561.

(11) Franco, I; Tretiak, S. Electron-vibrational dynamics of photoexcited polyfluorenes. J. Am. Chem. Soc. 2004, 126, 12130-12140.

(12) Marcon, V.; van der Vegt, N.; Wegner, G.; Raos, G. Modeling of molecular packing and conformation in oligofluorenes. J. Phys. Chem. B. 2006, 110, 5253-5261.

(13) Kilina, S.; Batista, E.R. ; Yang, P.; Tretiak, S.; Saxena, A.; Martin, R.L. ; Smith, D.L. Electronic structure of selfassembled amorphous polyfluorenes. ACS Nano 2008, 2, 1381-1388.

(14) Knaapila, M.; Stepanyan, R.; Lyons, B.P.; Torkkeli, M.; Monkman, A.P. Towards General Guidelines for Aligned, Nanoscale Assemblies of Hairy-Rod Polyfluorene. Adv. Funct. Mater. 2006, 16, 599-609.

(15) Fron, E.; Deres, A.; Rocha, S.; Zhou, G.; Müllen, K.; De Schryver, F.C.; Sliwa, M.; Uji-i, H.; Hofkens, J.; Vosch, T. Unraveling excited-state dynamics in a polyfluorene-perylenediimide copolymer. J. Phys. Chem. B. 2010, 114, 12771286.

(16) Kim, J. S. ; Lu, L.; Sreearunothai, P.; Seeley, A.; Yim, K.H. ; Petrozza, A.; Murphy, C. E. ; Beljonne, D.; Cornil, J.; Friend, R. H. Optoelectronic and charge transport properties at organic-organic semiconductor interfaces: comparison between polyfluorene-based polymer blend and copolymer. J Am Chem Soc. 2008, 130, 13120-13131. 
(17) Chunwaschirasiri, W.; Tanto, B.; Huber, D. L.; Winokur, M. J. Chain conformations and photoluminescence of poly(di-n-octylfluorene). Phys. Rev. Lett. 2005, 94, 107402.

(18) Clark, J.; Nelson, T.; Tretiak, S.; Cirmi, G.; Lanzani, G. Femtosecond Torsional Relaxation. Nat. Phys. 2012, 8, 255-231.

(19) Nelson, T., Fernandez-Alberti, S., Chernyak, V., Roitberg, A. E. Tretiak, S.. Nonadiabatic excited-state molecular dynamics modeling of photoinduced dynamics in conjugated molecules. J. Phys. Chem. B 2011, 115, 5402-5414.

(20) Nelson, T; Fernandez-Alberti, S; Roitberg, A.E.; Tretiak, S. Nonadiabatic excited-state molecular dynamics: modeling photophysics in organic conjugated materials. Acc Chem Res. 2014, 47,1155-1164.

(21) Car, R.; Parrinello, M. Unified Approach for Molecular Dynamics and Density-Functional Theory. Phys. Rev. Lett. 1985, 55, 2471.

(22) Frank, I; Hutter, J; Marx, D; Parrinello, M. Molecular dynamics in low-spin excited states. J. Chem Phys. 1998, $108,4060-4069$

(23) Vreven, T.; Bernardi, F.; Garavelli, M.; Olivucci, M.; Robb, M. A.; Schlegel, H. B. Ab Initio photoisomerization dynamics of a simple retinal chromophore model. J. Am. Chem. Soc. 1997, 119, 12687- 12688.

(24) Tavernelli, I; Röhrig, U.F.; Rothlisberger, U. Molecular dynamics in electronically excited states using timedependent density functional theory. Mol. Phys. 2005, 103, 963-981.

(25) Doltsinis, N.L.; Marx, D. Nonadiabatic Car-Parrinello Molecular Dynamics. Phys. Rev. Lett. 2002, 88, 166402.

(26) Doltsinis, N; Marx, D. First Principles Molecular Dynamics Involving Excited States and Nonadiabatic Transitions. J. Theor. Comput. Chem. 2002, 1, 319-349.

(27) Curchod, B.F.E.; Martínez, T.J. Ab initio nonadiabatic quantum molecular dynamics. Chem. Rev. 2018, 118, $3305-3336$

(28) Hollas, D.; Šištík, L.; Hohenstein, E.G.; Martínez, T.J.; Slavíček, P. Nonadiabatic ab initio molecular dynamics with the floating occupation molecular orbital-complete active space configuration interaction method. J. Chem. Theory Comput. 2018, 14, 339-350.

(29) Tully, J. C.; Preston, R.K. Trajectory surface hopping approach to nonadiabatic molecular collisions: the reaction of $\mathrm{H}^{+}$with $\mathrm{D}_{2}$. J. Chem. Phys. 1971, 55, 562-572.

(30) Tully, J. C. Molecular dynamics with electronic transitions. J. Chem. Phys. 1990, 93, 1061-1071.

(31) Schmidt, J. R.; Parandekar, P. V.; Tully, J. C. Mixed quantum-classical equilibrium: Surface hopping. J. Chem. Phys. 2008, 129, 044104.

(32) Tapavicza, E.; Tavernelli, I; Rothlisberger, U. Trajectory surface hopping within linear response Time-Dependent Density-Functional Theory. Phys. Rev. Lett. 2007, 98, 023001.

(33) Tapavicza, E.; Bellchambers, G. D.; Vincent, J. C.; Furche, F. Ab initio non-adiabatic molecular dynamics. Phys. Chem. Chem. Phys. 2013, 15, 18336-18348.

(34) Nelson, T.; Fernandez-Alberti, S; Chernyak, V; Roitberg, A.E.; Tretiak S. Nonadiabatic excited-state molecular dynamics: numerical tests of convergence and parameters. J Chem Phys. 2012,136, 054108

(35) Hamelberg, D.; Mongan, J.; McCammon, J. A. Accelerated molecular dynamics: a promising and efficient simulation method for biomolecules. J. Chem. Phys. 2004, 120, 11919-11929.

(36) Pierce, L. C. T.; Markwick, P. R. L.; McCammon, J. A.; Doltsinis, N. L. Accelerating chemical reactions: exploring reactive free-energy surfaces using accelerated ab initio molecular dynamics. J. Chem. Phys. 2011, 134 174107.

(37) Ortiz-Sánchez, J.M.; Bucher, D; Pierce, L.C.T.; Markwick, P.R.L.; McCammon, J.A. Exploring the Photophysical Properties of Molecular Systems Using excited state accelerated ab initio molecular dynamics. $J$ Chem Theory Comput. 2012, 8, 2752-2761.

(38) Pietropaolo, A.; Wang, Y.; Nakano T. Predicting the switchable screw sense in fluorene-based polymers. Angew. Chem Int Ed, 2015, 54, 2688-2692.

(39) Pietropaolo, A.; Branduardi, D.; Bonomi, M.; Parrinello, M. A chirality-based metrics for free-energy calculations in biomolecular systems. J. Comput. Chem. 2011, 32, 2627-2637.

(40) Körzdörfer, T.; Brédas, J. L. Organic electronic materials: recent advances in the DFT description of the ground and excited states using tuned range-separated hybrid functionals. Acc Chem Res. 2014, 47, 3284-3291.

(41) Chen, X.K.; Tsuchiya, Y.; Ishikawa, Y.; Zhong, C.; Adachi, C.; Brédas, J.L. A New Design Strategy for Efficient Thermally Activated Delayed Fluorescence Organic Emitters: From Twisted to Planar Structures. Adv Mater. 2017, 29, 1702767.

(42) Green, M. M.; Peterson, N. C.; Sato, T.; Teramoto, A.; Cook, R.; Lifson, S. Science 1995, 268, 1860-1866.

(43) Lifson, S.; Andreola, C.; Peterson, N. C.; Green, M. M. J. Am. Chem. Soc. 1989, 111, 8850-8858.

(44) Marques, M.A.L.; Gross, E.K.U. Time-dependent density functional theory. Annu. Rev. Phys. Chem. 2004, 55, 427-455.

(45) Barducci , A.; Bussi, G.; Parrinello, M. Well-Tempered Metadynamics: a smoothly converging and tunable freeenergy method. Phys. Rev. Lett. 2008, 100,020603.

(46) Laio, A.; Parrinello, M. Escaping free-energy minima. Proc. Natl. Acad. Sci. USA. 2002, 99, 12562-12566.

(47) Branduardi, D; Bussi, G.; Parrinello, M. Metadynamics with adaptive Gaussians. J. Chem. Theory Comput. 2012 $8,2247-2254$

(48) Imamura, A.; Hoffmann, R. Electronic structure and torsional potentials in ground and excited states of biphenyl, fulvalene, and related compounds. J. Am. Chem. Soc. 1968, 90, 5379-5385. 